\title{
DIAGNÓSTICO DIFERENCIAL DOS TREMORES
}

\author{
JAMES PITÁGORAS DE MATTOS *
}

\begin{abstract}
RESUMO - Baseado na revisão da literatura, o autor refere-se aos tremores normal (fisiológico) e anormal (patológico). Analisa particularmente os patológicos, dividindo-os em de repouso e de ação. Com os elementos semiológicos estabelece o diagnóstico diferencial entre os tremores parkinsoniano, essencial severo, rubral, tardio, postural, cinético e os de posição e de ação específica, entre outros.
\end{abstract}

PALAVRAS-CHAVE: tremores, repouso, ação.

\section{Differential diagnosis of tremors}

ABSTRACT - After the review of the literature the author presents the semiologic basis for the differential diagnosis of rest and action tremors. The parkinsonian, severe essential, rubral, tardive, postural, kinetic and the task or position-specific tremors are mainly analysed.

KEY WORDS: tremors, rest, action.

Há nove anos atrás, Donald Calne ${ }^{1}$,revendo os manuscritos encontrados no palácio de Windsor, concluiu que Leonardo da Vinci (1452-1514), provavelmente já conhecia a doença de Parkinson, pois, é de sua autoria a seguinte citação: “... acho que você verá moverem-se os paralíticos, os friorentos e os prostrados; os seus membros tremulam com a cabeça e com as mãos, sem a permissão da alma; qual alma, que com todas as suas forças, não poderá impedir a esses membros que não tremam." ${ }_{1,2}$. Ainda sob o ponto de vista histórico, outras citações, tais como as de Shakespeare em Henrique VI, no IV ato, na cena I: “...veremos se essa cabeça fica quieta na ponta de uma vara. Levai-o e logo decapitai-o," ${ }^{1,2}$. Segundo Calne ${ }^{1}$, essas referências, entre outras, contrariam a hipótese de que a doença de Parkinson tenha surgido com a era industrial, embora nesses dois textos não constem elementos suficientes para o diagnóstico diferencial com o tremor essencial.

Entende-se por tremor o movimento involuntário de caráter oscilatório e rítmico, mais ou menos regular, produzido por contrações sincrônicas ou alternantes de músculos antagonistas ${ }^{3-6}$. Nesse conceito, o caráter oscilatório e rítmico praticamente diferencia o tremor de outros movimentos involuntários; também, separa o tremor parkinsoniano do essencial, pois, o primeiro é eletromiograficamente determinado por atividade alternante de músculos agonistas e antagonistas, enquanto o segundo, por atividade de músculos sincrônicos ${ }^{4}$.

Basicamente, há duas categorias de tremores: o normal (fisiológico) e o anormal (patológico). O tremor normal ocorre na frequência de $8-13 \mathrm{~Hz}$ e uma das explicações para sua existência é a de que seria o reflexo do balistocardiograma, isto é, da vibração passiva dos tecidos do corpo produzida pela atividade mecânica do coração. Não é habitualmente percebido, podendo, no entanto, ser

Estudo realizado no Serviço de Neurologia do Hospital Universitário Clementino Fraga Filho da Universidade Federal do Rio de Janeiro (UFRJ) (Chefia: Prof Sérgio Novis):* Professor Adjunto e Chefe do Setor de Disturbios do Movimento. Aceite: 26-fevereiro-1998.

Dr. James Pitágoras de Mattos - Av. São Sebastião, 165/101 - 22291-070 Rio de Janeiro RJ - Brasil. 
exacerbado por certas condições, tais como: situações de medo e ansiedade, distúrbios metabólicos (hipertireoidismo , hipoglicemia), exercícios físicos, retirada de álcool e uso de certas drogas (lítio, prednisona). Nessas condições há aumento do débito cardíaco, sendo exemplos de exacerbações do tremor fisiológico ${ }^{3,4,6,8}$. O tremor patológico é bem mais lento, ocorrendo na frequência de 4-7 Hz (a metade do normal). Atinge certos grupos como partes distais dos membros, cabeça, língua, mento, corda vocal e , raramente, o tronco ${ }^{3,4,6}$.

No que se refere ao diagnóstico diferencial dos tremores anormais ou patológicos, seguiremos a classificação fenomenológica de $\operatorname{Jankovic}^{6}$ (Tabela), que os divide em três grupos principais: tremor de repouso; tremor de ação; miscelânea e outros movimentos rítmicos.

\section{TREMORES DE REPOUSO}

O tremor de repouso é aquele que ocorre quando o segmento corporal está relaxado ou fora da ação da gravidade e que desaparece com ato motor voluntário. Costuma ser encontrado na doença de Parkinson (que é a causa principal), no parkinsonismo plus, no parkinsonismo heredodegenerativo e no parkinsonismo secundário. Além das quatro categorias de parkinsonismo, ocorre ainda no tremor essencial severo, no tremor rubral, no tremor tardio, na miorritmia e no "spasmus nutans".

Tremor Parkinsoniano - Causa principal de tremor de repouso, registra-se na frequência típica de 3-6 Hz, mais observado em uma ou em ambas as mãos, menos notado no mento, nos lábios, na língua e nos pés. Eletromiograficamente caracteriza-se por resultar de atividade alternante de músculos agonistas e antagonistas. A flexão-extensão dos dedos combinada com a adução-abdução do polegar produz o clássico tremor em "contar dinheiro"ou em "enrolar fumo"4-8.

Tremor essencial severo - Como é sabido, habitualmente o tremor parkinsoniano é de repouso e o tremor essencial é de ação (postural). No entanto, o tremor parkinsoniano pode ser também postural e o tremor essencial de repouso. O tremor essencial severo, condição na qual o movimento anormal exibe-se com acentuado aumento na sua amplitude, é o exemplo de tremor essencial que pode ocorrer em repouso. Não se sabe se esses pacientes têm doença de Parkinson associada ou se mais tardiamente desenvolverão outros sinais parkinsonianos ${ }^{6-8}$.

Tremor rubral - Devido à proximidade da lesão do núcleo rubro, criou-se esse termo inadequado. $\mathrm{O}$ tremor é grosseiro, lento $(3-5 \mathrm{~Hz})$, mais intenso com o movimento, mas, pode ser observado também em repouso. Costuma estar associado com ataxia do tipo cerebelar e oftalmoplegia do III nervo, configurando a síndrome de Benedikt $t^{3,4,6}$.

Tremor tardio - É observado em pacientes em uso crônico de neurolépticos. Pode ser de repouso ou de ação e se agrava com a retirada do neuroléptico e melhora com o emprego de drogas depletoras de dopamina, como, por exemplo, com a tetrabenazina. Pode vir acompanhado de outros movimentos anormais, tais como, acatisia, distonia e coréia ${ }^{6,9}$.

Miorritmia - Lembra muito o tremor parkinsoniano diferenciando-se, no entanto, pelas seguintes características: é mais lento $(2-3 \mathrm{~Hz})$, é bem mais de flexão-extensão do que de pronaçãosupinação e não apresenta outros sinais parkinsonianos. Alguns casos estudados com autópsia revelaram alterações vasculares ou degenerativas no tronco cerebral (principalmente na substância negra) e no cerebelo ${ }^{6}$.

"Spasmus nutans" - É uma condição muito rara, de natureza familiar, que ocorre antes de 1 ano de idade, caracterizada por inclinação rítmica da cabeça e nistagmo. Desaparece entre 1 e 2 anos de idade ${ }^{4,6}$.

\section{TREMORES DE AÇÃO}

O tremor de ação é aquele que ocorre quando há contração voluntária dos músculos, podendo ser subdividido em: postural, cinético, de posição e de ação específica e, ainda, isométrico ${ }^{4-7}$. 
Tabela. Classificação e diagnóstico diferencial dos tremores(adaptado de Jankovic,1992).

\section{TREMORES DE REPOUSO}

A. Doença de Parkinson

B. Outras síndromes parkinsonianas Atrofias de múltiplos sistemas Paralisia supranuclear progressiva Degeneração gangliônica cortico-basal

Parkinsonismo-demência-ELA de Guam Doença difusa do corpo de Lewy

Doenças heredo-degenerativas

Doença de Huntington

Atrofia palidal progressiva

Doença de Wilson

Neuro-acantocitose

Doença de Hallervorden-Spatz

Doença de Gerstmann-Strauler-Scheinker

Lipofuscinose ceróide

D. Parkinsonismo secundário

Tóxico: MPTP , CO, Mn, metanol, cianeto, CS2

Induzido por drogas:bloqueadoras dos receptores de dopamina,depletoras de dopamina

Vascular: múltiplos infartos, doença de Binswanger, "lower-body

Trauma: encefalopatia pugilística, lesão mesencefálica

Tumor e paraneoplasia

Infecção: pós-encefalítico, fungo, AIDS, PESA, doença de Creutzfeldt-Jakob

Metabólico: hipoparatireoidismo, degeneração hepática crônica

Hidrocefalia com pressão normal

E. Tremor essencial severo

F. Tremor rubral

G. Tremor tardio

H. Miorritmia

I. "Spasmus nutans"

II. TREMORES DE AÇÃO

A. TREMORES POSTURAIS

1. Tremor fisiológico

2. Tremor fisiológico exacerbado

a. induzido por stress: emoção, exercício, fadiga, ansiedade, febre

b. endócrino: hipoglicemia, tireotoxicose, feocromocitoma, adrenocorticosteróides

c. drogas: agonistas beta (teofilina, epinefrina), dopaminérgicas (levodopa, agonistas), estimulantes (anfetaminas), psiquiátricas (lítio, neurolépticos, tricíclicos), metilxantinas (café, chá), ácido valpróico, ciclosporina

d. toxinas: $\mathrm{Hg}, \mathrm{Pb}, \mathrm{As}, \mathrm{Bi}, \mathrm{Br}$, retirada de álcool

3. Tremor essencial

a. autossômico dominante

b. esporádico

4. Tremor postural associado a

a. distonia

b. parkinsonismo

c. mioclonia

d. neuropatia sensitivo-motora hereditária

5. Doença de Parkinson e outras síndromes parkinsonianas

6. Tremor tardio

7. Tremor rubral

8. Tremor hipotônico cerebelar

9. Tremor neuropático (doença do neurônio, neuropatia periférica, distrofia simpática reflexa)

B. TREMORES CINÉTICOS (intencional dinâmico e terminal)

1. Desordens cerebelares: esclerose múltipla, trauma, AVE, doença de Wilson, drogas e toxinas

2. Lesões mesencefálicas

C. TREMORES DE POSIÇÃO ESPECÍFICA E DE AÇÃO ESPECÍFICA

1. Escrita

2. Ortostático

3. Outros (ocupacionais: músicos, esportistas)

D. ISOMÉTRICO

III. TREMORES: MISCELÂNEA E OUTROS MOVIMENTOS RÍTMICOS
A. MIOCLONIA (palatal, oscilatória, asterix, mini-polimioclonus)
B. TREMOR DISTONICO
C. EPILEPSIA PARCIAL CONTÍNUA
D. NISTAGNO
E. CLONUS
F. FASCICULAÇÃO 
Tremor postural - É evidenciado durante a manutenção de uma determinada postura antigravitacional , como, por exemplo, quando os membros superiores são mantidos estendidos na frente do corpo. Costumam desaparecer com o relaxamento do referido segmento. Eletromiograficamente caracteriza-se por atividade rítmica sincrônica dos músculos agonistas e antagonistas. Na prática neurológica o exemplo mais comum de tremor postural é o tremor essencial (esporádico ou familiar) $\mathrm{O}$ tremor essencial é a condição que mais frequentemente se confunde com a doença de Parkinson. É um distúrbio monossintomático caracterizado por tremor fino, rápido, na frequência de 5-7 Hz. Ocorre em qualquer idade, sendo mais comum após os 40 anos, em qualquer parte do corpo, sendo mais frequente nas mãos, logo seguida da cabeça; piora com as emoções, com a fadiga e com o avançar da idade; melhora consideravelmente com a ingestão de bebidas alcoólicas e com drogas betabloqueadoras, assim como com primidona ${ }^{3-8,10,11}$.

Tremor cinético - É aquele que ocorre durante a realização de determinado ato motor.Pode ser inicial, dinâmico ou final quando surge, respectivamente, no início, no curso ou no final do ato motor. Está tipicamente associado com lesões ou doenças do cerebelo ${ }^{3-7}$. A frequência é de 3-4 Hz. O termo"titubeio" refere-se a oscilação rítmica da cabeça ou do tronco, presumivelmente causado por hipotonia dos músculos axiais ${ }^{4}$.

Tremores de posição específica e de ação específica - Podem ser:

a) de posição específica - Quando o tremor ocorre em certas posturas como, por exemplo, com a xícara ou com copo próximo da boca, ou quando na posição bípede ${ }^{5,711}$ Nesse último caso, denomina-se de tremor ortostático, curiosa condição descrita por Heilman, em 1984, que se caracteriza pelo surgimento de tremor nos membros inferiores somente quando o paciente adota a posição em pé. Diminui com a marcha e desaparece quando o paciente senta-se ou quando reclina-se. Sentado ou reclinado o tremor poderá ser provocado por forte contração da perna contra resistência ${ }^{3}$.

b) de ação específica - Quando o tremor ocorre em certas tarefas, como por exemplo, ao escrever (tremor da escrita primário), ao falar ou ao cantar (tremor vocal) $)^{5,711,12}$.

Tremor isométrico - É aquele que aparece durante a contração muscular voluntária mantida contra determinado objeto fixo $0^{5,7}$.

\section{MISCELÂNEA}

Finalmente, em quase todas as classificações, há um grupo que não pertence exatamente aos anteriores e que, por isso, são colocados separadamente - é o grupo miscelânea e outros movimentos rítmicos. São exemplos a mioclonia (que por vezes pode ser rítmica), o tremor distônico (de negação), a epilepsia parcial continua, o nistagmo, o clono, as miofasciculações, os calafrios, o "head bobbing" e o sinal de Musset da insuficiência aórtica ${ }^{5,7}$.

\section{REFERÊNCIAS}

1. Calne D. Did Leonardo describe Parkinson's disease? (Letter) N Engl J Med1989;320:594

2. Werneck Neto ALS. Fatores de risco para o parkinsonismo. Tese de Mestrado, Universidade Federal do Rio de Janeiro, 1996.

3. Adams R, Victor M. Principles of neurology (Tremor, myoclonus, spasms and tics). 5Ed. Mc Graw-Hill, 1993:83-98.

4. Fahn S.Differential diagnosis of tremors.Med Clin North Amer 1972,56;1363-1375.

5. Horta W. Tremor. XVII Congresso Brasileiro Neurologia1996, Curso Distúrbios Movimento,Curitiba:1996:20.

6. Jankovic J. Tremors:diagnosis and treatment.American Academy of Neurology,1992, Movement Disorders Course, San Diego1992:13-37.

7. Jankvic J.The extrapyramidal disorders. In Bennett C, Plum F (eds.) Cecil textbook of medicine. 20 Ed; Philadelphia:Saunders, 1996:2047.

8. Mattos JP. Diagnostico diferencial. In Meneses MS, Teive HAG (Eds). Doença de Parkinson: aspectos clínicos e cirúrgicos. Rio de Janeiro: Guanabara Koogan, 1996;74-82.

9. Gelenberg AJ, Jefferson JW.Lithium tremor.J Clin Psychiatry 1995;56:283-287.

10. Elbe RJ.The role of aging in the clinical expression of essential tremor. Exp Gerontol,1995;30:337-347.

11. Hallet M. Classification and treatment of tremor. JAMA 1991;266:1115-1117.

12. Bain PG, Findley LJ, Britton TC, et al.. Primary writing tremor. Brain 1995;118:1461-1472. 